\title{
Direct Comparison of Various Gaseous Secondary Electron Detectors in the Variable Pressure Scanning Electron Microscope
}

\author{
Matthew R. Phillips and Scott W. Morgan \\ Microstructural Analysis Unit, University of Technology, Sydney Broadway NSW Australia 2007
}

The conventional Everhart-Thornely scintillation-photomultiplier secondary electron (SE) detector cannot function at elevated pressures due to the high voltage $(\sim+12 \mathrm{kV})$ involved in its operation. As a result, SE imaging in the variable pressure scanning electron microscope (VPSEM) has required the development of a new generation of SE detectors that operate under low vacuum conditions. To date, three different methods have been devised to measure the secondary electron (SE) emission signal in a VPSEM. Each of these approaches involves the excitation of the chamber gas by the placement of a low voltage $(<+1000 \mathrm{~V})$ positively biased electrode in the vicinity of the specimen. A SE image can be obtained by measuring the current induced in either the positive electrode (the gaseous secondary electron detector) or the grounded stage (the ion current detector) or via a photomultiplier that detects light emission from the gas (the gas luminescence detector). In this work, the performance of each of these three low vacuum SE detector types has been compared under identical operating conditions using a Zeiss Supra 55VPSEM and FEI XL30 ESEM.

A typical gas gain curve measured from a conductive specimen as a function of pressure, $\mathrm{P}$, using an ion current detector is shown in Figure 1. The initial increase in the gain results from the decrease in the ionization mean free path with increasing $\mathrm{P}$. At higher $\mathrm{P}$, the gain falls as the mean free path becomes too short for electrons to acquire sufficient energy to ionize the imaging gas. However, no corresponding decrease in gain is observed in the gas amplification curve for a gas luminescence detector using equivalent collection parameters (Fig. 2) as light emission can still be excited well below the gas ionization energy via multiple low energy absorption processes. In addition, the lower luminescence excitation level was found to diminish gas the impact of amplification quenching due to the accumulation of positive ions (the space charge effects) when imaging insulating specimens (Figure 3 and 4).

SE images were collected from both insulating and conductive specimens at different scan speeds using the various VPSEM SE detectors types under identical conditions and a range of chamber gases (water vapor, nitrogen and argon). With conductive samples at high scan speeds, the dark and bright streaks in the direction of scan that are present in GSED (Figure 5) and ICD images (not shown) are absent in the gas luminescence image (Figure 6). This observation can be accounted for by the high bandwidth of the photomultiplier tube employed in the gas luminescence detector compared with read times involved in measuring the induced current from the signal collection electrodes. However, all three low vacuum detectors produce anomalous SE contrast when imaging regions of high SE emission in insulating specimens over a wide range of scan speeds (not shown), confirming the existence of long-lived metastable excited states during the light emission process for imaging gases used in this study. Based on the results of these studies, the merits and limitations of the current low vacuum SE detector technologies will be discussed. 


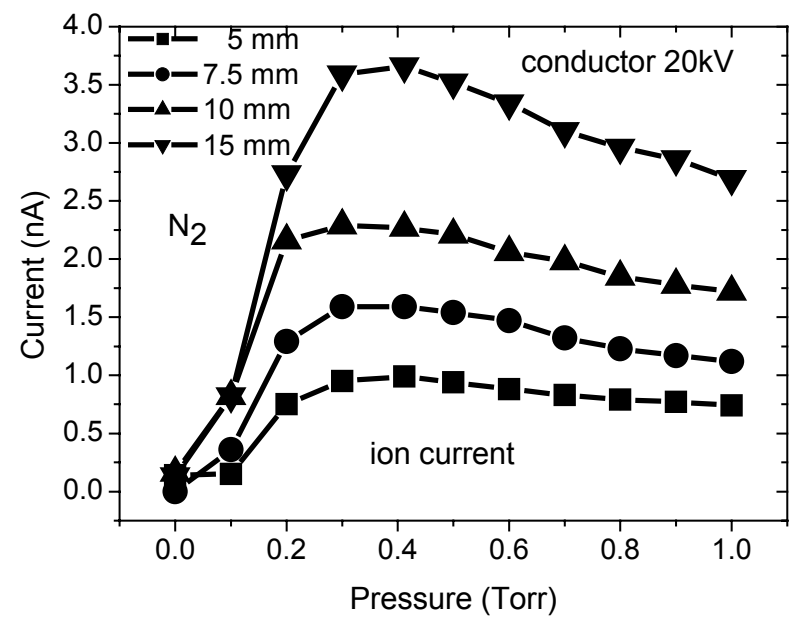

Figure 1

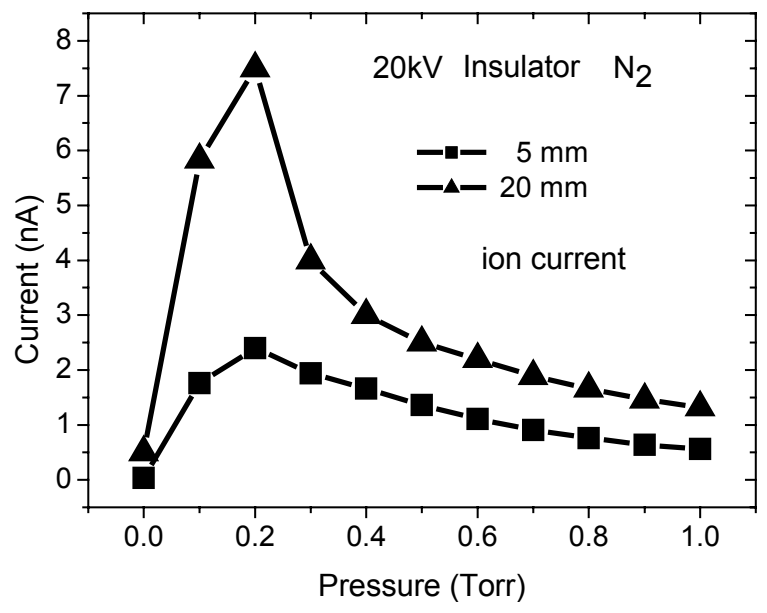

Figure 3

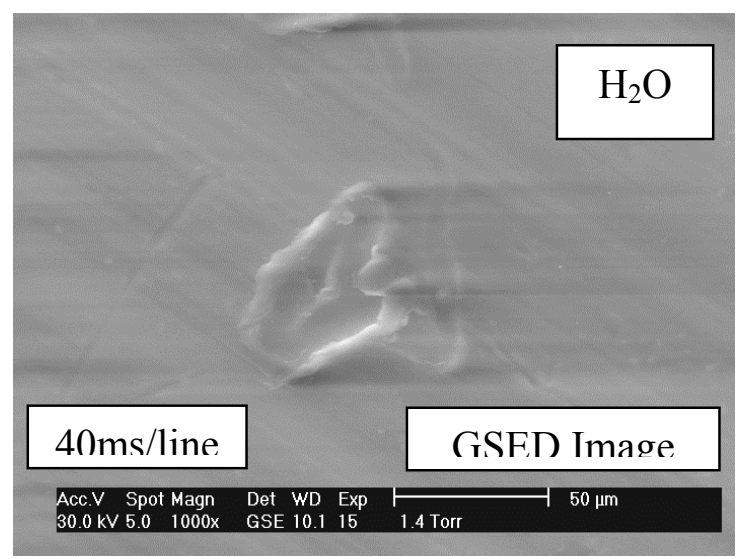

Figure 5

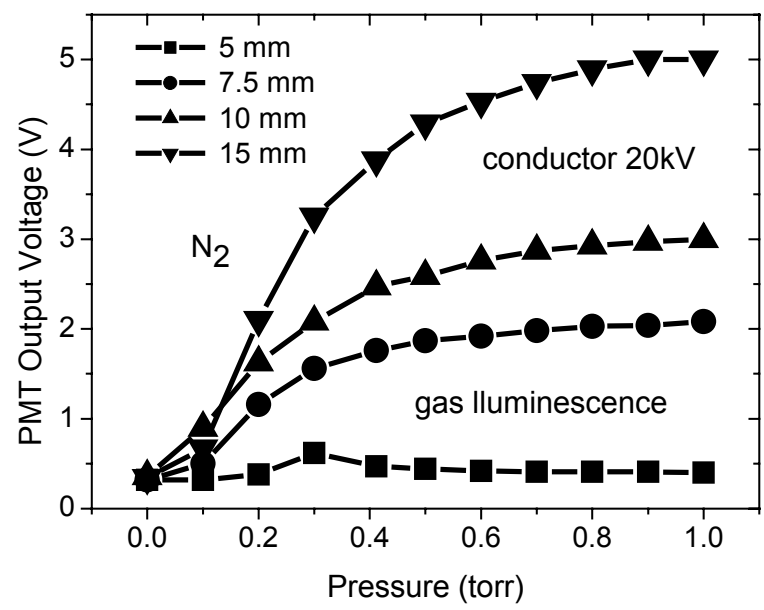

Figure 2

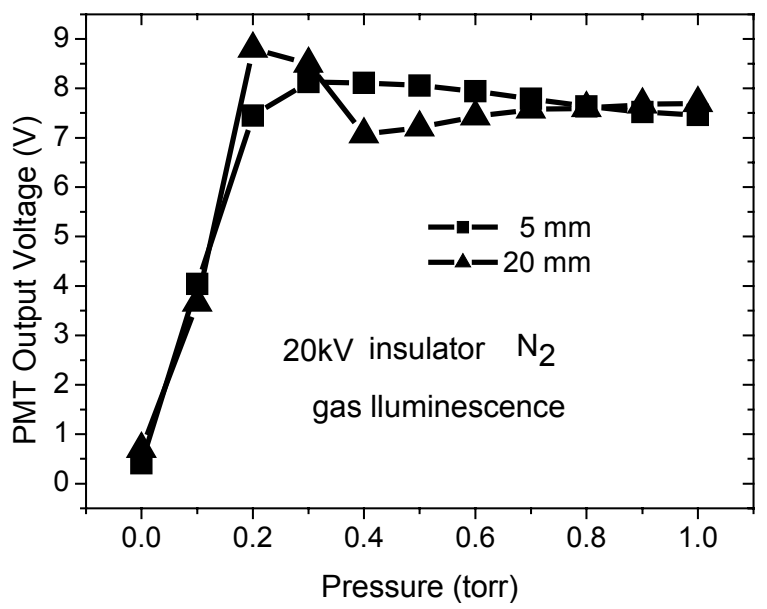

Figure 4

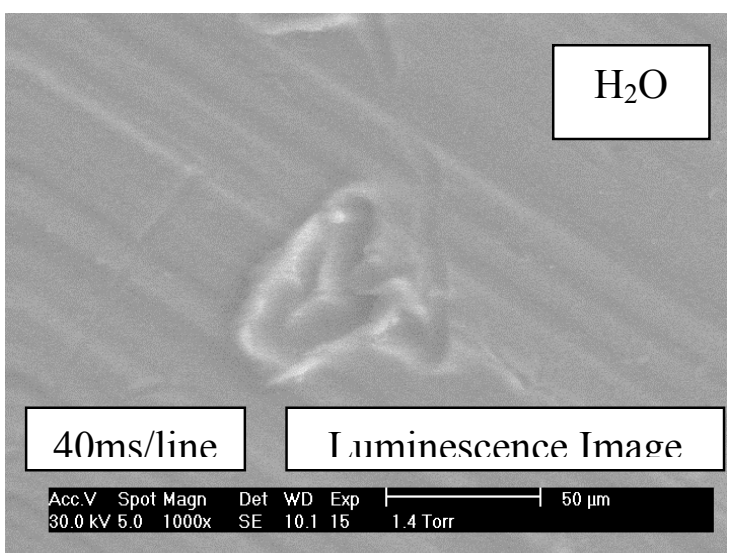

Figure 6 